\title{
Generation of electron vortices using nonexact electric fields
}

\author{
Amir H. Tavabi $\odot,{ }^{1, *}$ Hugo Larocque, ${ }^{2}$ Peng-Han Lu, ${ }^{1}$ Martial Duchamp, ${ }^{1,3}$ Vincenzo Grillo, ${ }^{4}$ \\ Ebrahim Karimi $\odot,{ }^{2,5, \dagger}$ Rafal E. Dunin-Borkowski, ${ }^{1}$ and Giulio Pozzi ${ }^{1,6}$ \\ ${ }^{1}$ Ernst Ruska-Centre for Microscopy and Spectroscopy with Electrons and Peter Grünberg Institute, \\ Forschungszentrum Jülich, 52425 Jülich, Germany \\ ${ }^{2}$ Department of Physics, University of Ottawa, 25 Templeton Street, Ottawa, Ontario K1N 6N5, Canada \\ ${ }^{3}$ School of Materials Science and Engineering, Nanyang Technological University, 50 Nanyang Avenue, Singapore 639798, Singapore \\ ${ }^{4}$ CNR Istituto Nanoscienze, Centro S3, Via G. Campi 213/a, I-41125 Modena, Italy \\ ${ }^{5}$ Department of Physics, Institute for Advanced Studies in Basic Sciences, 45137-66731 Zanjan, Iran \\ ${ }^{6}$ Department of Physics and Astronomy, University of Bologna, viale B. Pichat 6/2, 40127 Bologna, Italy
}

(Received 17 August 2018; revised manuscript received 12 June 2019; accepted 13 November 2019; published 21 February 2020)

\begin{abstract}
Vortices in electron beams can manifest several types of topological phenomena, such as the formation of exotic structures or interactions with topologically structured electromagnetic fields. For instance, the wave function of an electron beam can acquire a phase vortex upon propagating through a magnetic monopole. In practice, this provides a convenient method for generating electron vortex beams, yet it is very limited by the structural integrity of devices used for such purposes. Here, we show how an electric field must be structured in order to achieve a similar effect. We find that closed but not exact electric fields can produce electron vortex beams. We proceed by fabricating a versatile, robust, and near-obstruction-free device that is designed to approximately produce such fields and we systematically study their influence on incoming electron beams. With such a single device, electron vortex beams that are defined by a wide range of topological charges can be produced by means of a slight variation of an applied voltage. For this reason, this device is expected to be important in applications that rely on the sequential generation and manipulation of different types of electron vortices.
\end{abstract}

DOI: 10.1103/PhysRevResearch.2.013185

Vortices can generally be described as stagnant points surrounded by a form of coiling motion. These entities can, for instance, occur within complex fields, such as those describing the wave function of a quantum system or scalar optical waves. In such systems, vortices manifest themselves as singular points of the wave field's phase, i.e., points around which the phase varies by an integer multiple $\ell$ of $2 \pi$, where $\ell$ is referred to as the topological charge of the singularity [1-3]. In many cases, the presence of singularities in wave fields can lead to exotic forms of topological or geometric phenomena. For instance, the presence of a polarization singularity in a tightly focused optical wave can lead to the formation of a Möbius strip [4], while optical beams with carefully structured phase and polarization singularity distributions can result in the formation of knots [5,6]. Other types of singular behavior can occur when matter waves of charged particles interact with structured electromagnetic fields. For instance, an electron beam propagating through a magnetic monopole

\footnotetext{
*a.tavabi@fz-juelich.de

†ekarimi@uottawa.ca
}

Published by the American Physical Society under the terms of the Creative Commons Attribution 4.0 International license. Further distribution of this work must maintain attribution to the author(s) and the published article's title, journal citation, and DOI. is known to acquire a vortex with a topological charge that is proportional to the strength of the monopole [7,8]. The impartment of this vortex arises directly from the electron's charge, in conjunction with the topological structure of the magnetic field. In practice, the latter is often approximated by the tip of a magnetic needle $[9,10]$. When this vortex is located at the center of the beam, the electron effectively acquires $\hbar \ell$ units of orbital angular momentum (OAM), where $\hbar$ is the reduced Planck constant [11]. In conjunction with the electron's charge, the presence of a vortex also causes the electron to acquire a magnetic dipole moment $\ell \mu_{B}$, where $\mu_{B}$ is the Bohr magneton. This magnetic property makes OAM-carrying electrons desirable in materials science, as it allows them to be employed as nanoscale magnetic probes [12-14]. For this reason, magnetic needles offer an appealing alternative to other types of electron beam-shaping methods, such as diffractive holograms $[12,15,16]$ and refractive phase masks $[17,18]$, which are both able to generate electron vortex beams. However, these devices have some technological constraints, including limited spatial resolution and an inability to generate electron vortices that are defined by arbitrary topological charges with a single device. For the specific case of magnetic needles, their magnetic nature prevents them from being positioned in the back focal plane of a magnetic lens and therefore to be used in electron imaging techniques that are analogous to optical spiral phase contrast microscopy [19,20]. Finally, the physical endurance of needles currently prevents 
them from generating electrons that are defined by larger values of OAM that are desirable in applications that rely on the stronger magnetic dipole moment that is carried by these electrons [14,21]. In this article, we discuss how an electric field can be shaped to impart a phase vortex on an electron beam. The requirement to achieve such an effect relies deeply on the nature of the topology of electric fields. We present an implementation of a device based on a recently proposed design [22] that can be seen as an electric counterpart to a magnetic needle and demonstrate how its electrical properties, in conjunction with its structural durability, can be used to generate electrons that carry a much broader range of tunable OAM values than those generated using magnetic needles.

In order to acquire OAM, electron waves need to acquire an azimuthally dependent phase that results in the addition of an $\exp (i \ell \varphi)$ term to their mathematical formulation, where $\varphi$ is the transverse azimuthal coordinate. Such phases can be acquired by making the electron propagate through a potential whose action induces a structured phase shift $[15-18,23]$. The phase $\theta$ acquired by an electron wave upon propagation through a system that is characterized by the presence of an electromagnetic field can be expressed in the form [11]

$$
\theta=\underbrace{\frac{1}{\hbar} \int(\mathbf{p} \cdot d \mathbf{r}-\mathcal{E} d t)}_{\text {Dynamic Phase }}+\underbrace{\frac{e}{\hbar} \int \mathbf{A} \cdot d \mathbf{r}}_{\text {Dirac Phase }}+\underbrace{\int \mathcal{A} \cdot d \mathbf{R}}_{\text {Berry Phase }} .
$$

The first term in this equation accounts for the dynamical phase acquired by the electron, where $\mathbf{p}$ is the electron's kinetic momentum, $\mathcal{E}=p^{2} / 2 m+e \Phi-\boldsymbol{\mu} \cdot \mathbf{B}$ is its energy, $m$ is its mass, $e$ is its charge, $\Phi$ is the field's scalar potential, $\boldsymbol{\mu}$ is the electron's magnetic dipole moment, and $\mathbf{B}$ is the magnetic field. This phase is typically used in the holographic generation of OAM-carrying electrons, given that such methods rely on devices which have a mean inner potential that affects both the energy and the momentum of propagating electrons [12,15-18]. The second term is the Dirac phase, where $\mathbf{A}$ is the field's vector potential. It is responsible for the impartment of OAM onto electrons propagating through a magnetic monopole [8] and other phenomena such as the AharonovBohm effect [24]. The last term represents the Berry phase, where $\mathcal{A}$ is the Berry curvature associated with an adiabatically varied parameter $\mathbf{R}$. Though this phase is known to be affected by electromagnetic fields [11], we reserve the treatment of its contribution to our device's performance for future work.

The OAM acquired by an electron beam exposed to a magnetic monopole is known to arise from the relative Dirac phase attributed to propagation along different transverse azimuthal angles. As depicted schematically in Fig. 1(a), this phase is proportional to the magnetic flux going through a surface bounded by the path taken by an electron and a reference path at $\varphi=0[3,9]$, which is proportional to the $\varphi$ coordinate. A more realistic treatment of this calculation involves the use of the field of a magnetic needle, which, as opposed to ideal monopoles, are readily realized in practice [9]. Geometrically speaking, this condition is enabled by the presence of a "2hole" at the origin of our coordinate system. More specifically, our magnetic field, which consists of a differential 2-form, is undefined at the origin and is thereby only formulated in
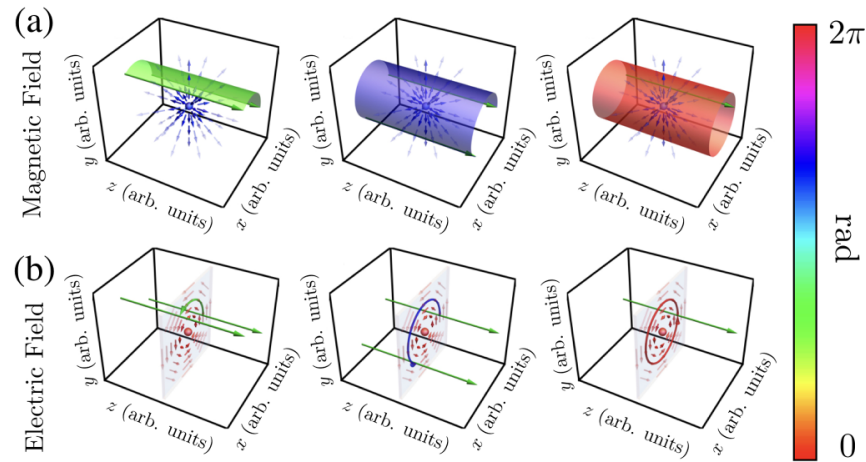

ڤั.

FIG. 1. Propagation of electrons through closed but not exact electromagnetic fields. (a) Surfaces used to calculate the relative azimuthal phase gained by electrons upon propagating through a magnetic monopole as investigated in Ref. [9]. (b) Lines used to calculate the relative azimuthal phase gained by electrons upon propagating through an azimuthally oriented electric field. Both the surfaces and the lines are colored based on the phase obtained from integrating the fields over them. The green arrows in the plots represent the two trajectories that are used as boundaries to calculate their relative azimuthal phases. In these plots, field strengths that were designed to add a phase of $\theta=\varphi$ to the electron beam were considered.

the manifold $\mathbb{R}^{3}-\{\mathbf{0}\}$. This construction prevents an arbitrary two-dimensional surface in the manifold to be deformed into another without crossing the hole, causing the magnetic field to be closed but not exact. This non-simply-connected space endowed with a "2-hole" gives rise to the radial nature of the magnetic field and therefore enables the impartment of a vortex on a propagating electron beam.

To obtain an electric field that can produce an electron vortex, one must first consider how electric fields differ from magnetic fields in terms of their influence on the phase of an electron wave, as indicated in Eq. (1). This process can conceptually be related to the electric field's topology. Namely, unlike magnetic fields, the differential forms that describe electric fields are 1-forms. For this reason, a closed yet not exact electric field can be constructed from the presence of a "1-hole." One can therefore expect that 1-holes can be used to impart a phase vortex onto an electron wave by arranging them in two-dimensional planes perpendicular to the wave's propagation. As displayed in Fig. 1(b), the phase resulting from the interaction between the electrons and the electric field should depend on lines enclosing the holes, i.e., a $1+1$-dimensional boundary, as opposed to the surfaces used in the case of the 2 forms, which consists of a $2+1$-dimensional boundary. This intuitive approach can be concretized by directly calculating the contribution of the electric field to the phase acquired by an electron from Eq. (1), i.e., $-(e / \hbar) \int \Phi d t$ [25]. Assuming the presence of a static electric field along with a paraxial configuration over which the electrons are propagating along the $z$ axis, the integration over time can be replaced by an integration along $z$. As a result, the acquired phase becomes $-\left(e m / \hbar p_{0}\right) \int \Phi d z$, implying that the potential $\Phi$ needs to be monotonic along $\varphi$ over a certain range $\Delta z$ to impart OAM to electrons. Such a requirement can also be seen by expressing the azimuthal domain of this potential with respect 

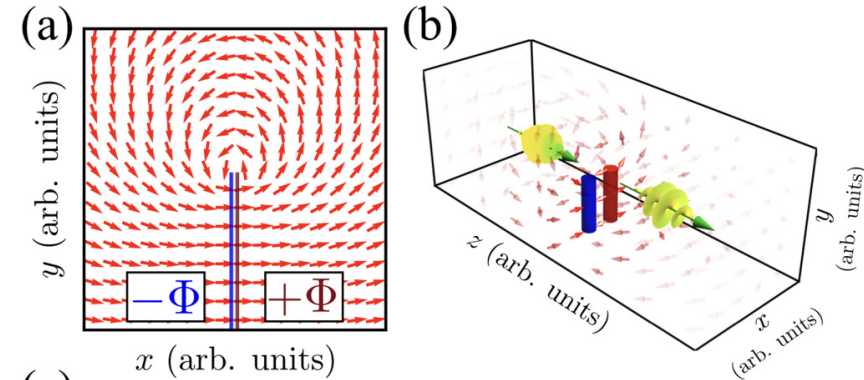

(c)

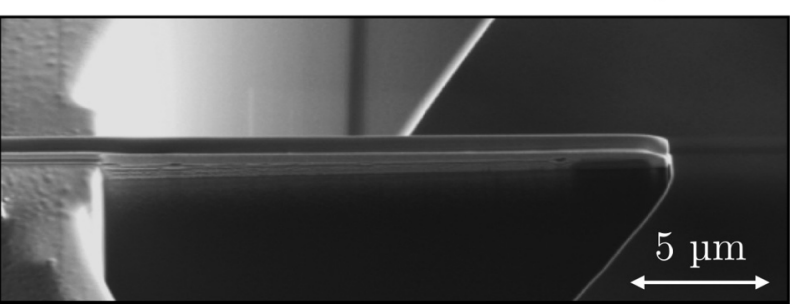

FIG. 2. Device generating a tunable, closed, but not exact electric field. Two submicron wires in close proximity with different electric potentials can effectively produce an azimuthally oriented electric field at their extremity. (a) Electric field resulting from such a configuration at the tips of both wires, which, represented as red and blue segments, are infinitely long and infinitely close to one another. (b) Device configuration required for the impartment of OAM onto an electron beam by means of such an electric field. The electric field is represented by red arrows, with a relative opacity that is dictated by its relative strength. (c) Scanning electron micrograph of a fabricated device, which consists of two 200-nm-wide and $15-\mu \mathrm{m}$-long nanowires separated by a $200-\mathrm{nm}$ gap, which lie on a silicon-nitride/silicon substrate.

to the azimuthal component of the electric field $E_{\varphi}$, i.e., $\Phi(\varphi, z)=-\int E_{\varphi}(\varphi, z) \rho d \varphi$. In order to impart a phase that increases linearly with azimuthal angle, an electric field with a constant azimuthal component is required. This requirement is shown schematically in Fig. 1(b). As initially postulated, the azimuthal nature of the electric field makes it not exact and constrains it to the manifold $\mathbb{R}^{2}-\{\mathbf{0}\}$ for a given $z$ value, requiring the presence of a " 1 -hole" at the origin. Moreover, the presence of the azimuthally varying phase ultimately relies on a line integral as opposed to a surface integral. In order to produce such an azimuthal electric field, one can adopt a dipole-like structure that locally displays fields with the geometry required to impart OAM onto electrons [22]. These local features can thereafter be enhanced by modifying the local geometry of the structure itself. As illustrated in Fig. 2(a), the tip of two elongated and extremely close charged rods allows for the generation of an almost perfectly azimuthal electric field. As in the case of magnetic needles [9,10], although the field of this structure is not strictly closed but nonexact, it still locally displays the features that are required to impart a vortex on the electron beam as shown in the experimental results of this work. By varying the charges on the two rods, the relative strength of the azimuthal electric field can be modified. Schematic diagrams of how such a device can be used to impart OAM onto incoming electrons are shown in Fig. 2(b). By varying the relative potential between the two rods, or equivalently the effective charge that they carry, the strength of the azimuthal electric field can be adjusted, thereby enabling the generation of electron vortices that are defined by a tunable amount of OAM. Because the strength of the electric field varies continuously as a function of relative potential, only discrete values of voltage applied between the two rods can lead to quantized azimuthal phase variations attributed to OAM.

This analysis may be confirmed analytically by considering the electric potential of a uniformly charged rod of length $a$ and charge density $K$ whose tip is centered at the origin [26]:

$$
\begin{aligned}
\Phi(x, y, z)= & \frac{K}{4 \pi \varepsilon_{0}}\left[\log \left(\frac{\sqrt{(a+y)^{2}+x^{2}+z^{2}}+a+y}{\sqrt{x^{2}+y^{2}+z^{2}}+y}\right)\right. \\
& \left.-\frac{a}{\sqrt{\left(x+x_{D}\right)^{2}+\left(y+y_{D}\right)^{2}+z^{2}}}\right]
\end{aligned}
$$

where the second term accounts for an arbitrarily positioned test charge in the $z=0$ plane. Knowing that the dynamic phase acquired by an electron propagating along the $z$ axis due to an electric field is given by $-\left(e m / \hbar p_{0}\right) \int \Phi d z$, then it follows from direct integration that this phase is given by

$$
\begin{aligned}
\theta(x, y)= & -\frac{m K e}{4 \pi \varepsilon_{0} \hbar p_{0}}\left[-(a+y) \log \left((a+y)^{2}+x^{2}\right)\right. \\
& +y \log \left(y^{2}+x^{2}\right)+2 a \\
& +2 x \arctan \left(\frac{y}{x}\right)-2 x \arctan \left(\frac{a+y}{x}\right) \\
& \left.+a \log \left(\left(x+x_{D}\right)^{2}+\left(y+y_{D}\right)^{2}\right)\right]
\end{aligned}
$$

Because the electric potential is additive, then one can obtain the phase attributed to our vortex generator by adding two phases attributed to oppositely charged shifted rods, i.e., $\theta(x-\alpha, y)-\theta(x+\alpha, y)$, where $2 \alpha$ is the separation between the two rods [22]. As demonstrated later, this phase locally displays the $\ell \varphi$ variations attributed to the presence of a vortex. If the length of this interaction is much smaller than the electron beam's diffraction length [3], then the electron wave function only acquires a phase shift. The wave function upon further propagation can then be calculated by solving the following Fresnel integral

$$
\psi(x, y, z) \propto \frac{p_{0}}{h z} \iint \psi\left(x^{\prime}, y^{\prime}, 0\right) \mathcal{K}\left(x, y, x^{\prime}, y^{\prime}\right) d x^{\prime} d y^{\prime},
$$

where $\psi\left(x^{\prime}, y^{\prime}, 0\right)$ is the wave function of the electron that is incident on the device and $\mathcal{K}\left(x, y, x^{\prime}, y^{\prime}\right)$ is a propagation Kernel given by

$$
\mathcal{K}\left(x, y, x^{\prime}, y^{\prime}\right)=e^{i\left(\frac{p_{0}}{2 h_{z}}\left[\left(x-x^{\prime}\right)^{2}+\left(y-y^{\prime}\right)^{2}\right]+\theta\left(x^{\prime}-\alpha, y^{\prime}\right)-\theta\left(x^{\prime}+\alpha, y^{\prime}\right)\right)} .
$$

If needed, a more detailed treatment of a beam propagating under the conditions imposed by an electron microscope can be achieved with more involved numerical methods [27].

In order to fabricate such a device, we adopted a fabrication procedure that involved the combined use of electron beam lithography and focused ion beam (FIB) milling. This approach enabled the fabrication of two 200-nm-wide and $15-\mu \mathrm{m}-$ long metallic wires separated by a $200-\mathrm{nm}$ gap, which were patterned lithographically onto a silicon nitride/silicon 
substrate. A semicircular opening with a radius of $15 \mu \mathrm{m}$ around the wires was then created using FIB milling. This design enables the substrate to be grounded to the microscope, while the wires are connected to an external voltage source, thereby preventing the formation of a short circuit between the wires. Given that the wires only span a bridge of $600 \mathrm{~nm}$ over the 30- $\mu \mathrm{m}$ circular opening, the device is almost obstructionfree, allowing higher transmission efficiency and reducing potential artefacts from scattering introduced by materialbased phase masks. A scanning electron micrograph of the device is shown in Fig. 2(c). Unlike previous electron vortex generators relying on electric fields [28], this device is tunable, provides the ability to generate a wide range of electron vortices, and, based on previous discussions, produces a field known to display the topological features required to do so. Furthermore, given that our device as a whole carries a net charge of zero, our electron wave does not acquire a lateral phase gradient from it, thereby making it more stable upon propagation.

The device's ability to impart a vortex on an electron was demonstrated by measuring it inside a transmission electron microscope (TEM, FEI Titan 60-300) equipped with a Schottky-type high-brightness field emission gun (FEI XFEG) and two electron biprisms. The microscope was operated at $300 \mathrm{kV}$ during the measurements. An electron biprism was used to form an interference region with a $1.5-\mu \mathrm{m}$ wide field of view. A 1.9-nm holographic interference fringe spacing was obtained by using a biprism voltage at $107 \mathrm{~V}$. Off-axis electron holography measurements obtained in this configuration were used to reconstruct the phase profile of electrons that had interacted with the device. Representative theoretical and experimental phase profiles are shown in Figs. 3(c) and 3(d) respectively and clearly display azimuthal variations attributed to the presence of a phase vortex.

As expected, the strength of these variations increases with that of the device's azimuthal electric field, which is tuned by means of applying a voltage difference between the two wires. In particular, voltages of \pm 5 and $\pm 10 \mathrm{~V}$ enable the generation of electron vortices that are defined by topological charges of $\ell \approx \pm 15$ and $\ell \approx \pm 30$, respectively. The presence of the vortices is also attested by the profile of the probability density function of the electrons' wave function. Defocused images recorded in the Fresnel domain provide a means of examining the latter quantity, given that they are acquired over many electrons and that the use of a larger defocus amplifies the small deflection of the electrons, thereby yielding a good reflection of the probabilistic nature of their wave function. The obtained theoretical and experimental images are shown in Figs. 3(e) and 3(f) respectively, for a series of different potentials applied to the two wires, as well as in Fig. 3(b), where no potential was applied. They display the expected presence of a null in the electron's probability density function located at the position of the phase vortex. The transverse extent of these nulls also displays the quintessential trend of increasing with the absolute value of the beam's topological charge. The phase and probability density profiles of the electron vortices are in good agreement with the expected phase profile given in Eq. (3) and numerical results based on Eq. (4). Slight discrepancies between the simulations and experiments arise primarily from minor details regarding how
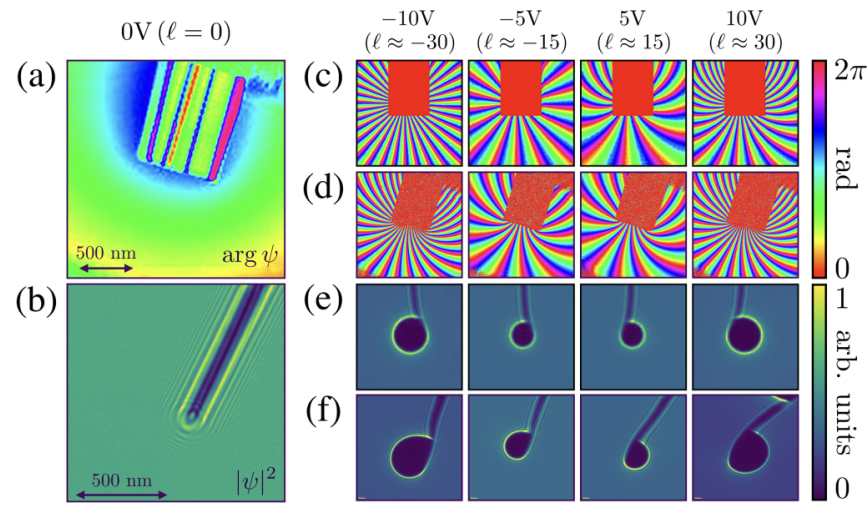

FIG. 3. Transverse spatial profile of electrons affected by the device. (a) TEM image of the device providing the phase, $\arg \psi$, of the electron beam when no voltage is applied to the device. (b) Corresponding TEM image of the propagated electron beam obtained from Fresnel imaging with a nominal defocus near $25 \mathrm{~mm}$. This image provides a measure of the probability density $|\psi|^{2}$ of the electron beam's wave function. Phase of an electron wave function having interacted with the device while the two wires were held at various potentials obtained from both (c) theory and (d) experiment. These phase profiles were reconstructed by means of off-axis electron holography defined by a fringe spacing of $1.9 \mathrm{~nm}$. Both the absolute value and the sign of the generated electron vortex beam's topological charge are observed to follow that of the applied voltage. Corresponding probability density functions of the electrons obtained from TEM images by means of Fresnel imaging experiments (e) based on theory and (f) obtained in experiments.

the device is fabricated. As the silicon nitride/silicon support of the wires becomes charged when it is being used, the presence of the support introduces additional contributions to the potential, akin to that of a biprism [22]. The finite length of the wires also contributes to discrepancies in the potential. These limitations can, however, be addressed with slight design modifications, such as the addition of static fields, as well as by ensuring that the wires can be biased independently.

In summary, we have demonstrated the tunable generation of electron vortex beams by means of closed but not exact electric fields. On a fundamental level, the structure of the field used in this generation scheme can be conceptually determined from the differential form describing electric fields and the need for 1-holes to generate the electron vortex. We were able to construct such fields by means of a nanofabricated device consisting of two wires held at different potentials. By adjusting the potential difference between the wires, we were able to adjust both the strength and the sign of the device's electric field, thereby enabling the generation of electron vortex beams defined by arbitrary topological charges. Besides demonstrating the relationship between the differential geometry of an electric field and the vortex content of an electron affected by it, our device holds significant promise for applications that rely on the sequential generation of electron vortex beams defined by different topological charges. Potential extensions of this device could include the use of larger numbers of electrodes to generate more complicated electron beam shapes-perhaps three-dimensional ones, especially if 
the electric fields can be modulated on a timescale over which the electron wave passes the device.

The authors acknowledge the support of the European Union's Horizon 2020 Research and Innovation Programme under Grant Agreement No. 766970 Q-SORT (H2020-FETOPEN-1-2016-2017). P.L. and R.E.D.B. acknowledge the support of the Deutsche Forschungsgemeinschaft for a Deutsch-Israelische Projektkooperation (DIP)
Grant. R.E.D.B. is grateful for funding from the European Research Council under the European Union's Seventh Framework Programme (FP7/2007-2013)/ERC Grant Agreement No. 320832. H.L. and E.K. acknowledge the support of Canada Research Chairs (CRC) and Ontario's Early Researcher Award (ERA). M.D. acknowledges the financial support of the Ministry of Education Academic Research Fund Tier 1 Grant No. RG101/17. The authors thank Maximilian Kruth for his help with the FIB work.
[1] K. Bliokh, I. Ivanov, G. Guzzinati, L. Clark, R. Van Boxem, A. Béché, R. Juchtmans, M. Alonso, P. Schattschneider, F. Nori et al., Phys. Rep. 690, 1 (2017).

[2] S. Lloyd, M. Babiker, G. Thirunavukkarasu, and J. Yuan, Rev. Mod. Phys. 89, 035004 (2017).

[3] H. Larocque, I. Kaminer, V. Grillo, G. Leuchs, M. J. Padgett, R. W. Boyd, M. Segev, and E. Karimi, Contemp. Phys. 59, 126 (2018).

[4] T. Bauer, P. Banzer, E. Karimi, S. Orlov, A. Rubano, L. Marrucci, E. Santamato, R. W. Boyd, and G. Leuchs, Science 347, 964 (2015).

[5] M. R. Dennis, R. P. King, B. Jack, K. O'Holleran, and M. J. Padgett, Nat. Phys. 6, 118 (2010).

[6] H. Larocque, D. Sugic, D. Mortimer, A. J. Taylor, R. Fickler, R. W. Boyd, M. R. Dennis, and E. Karimi, Nat. Phys. 14, 1079 (2018).

[7] P. A. M. Dirac, Phys. Rev. 74, 817 (1948).

[8] A. Fukuhara, K. Shinagawa, A. Tonomura, and H. Fujiwara, Phys. Rev. B 27, 1839 (1983).

[9] A. Béché, R. Van Boxem, G. Van Tendeloo, and J. Verbeeck, Nat. Phys. 10, 26 (2014).

[10] A. M. Blackburn and J. C. Loudon, Ultramicroscopy 136, 127 (2014).

[11] K. Y. Bliokh, Y. P. Bliokh, S. Savel'Ev, and F. Nori, Phys. Rev. Lett. 99, 190404 (2007).

[12] J. Verbeeck, H. Tian, and P. Schattschneider, Nature (London) 467, 301 (2010).

[13] V. Grillo, A. H. Tavabi, F. Venturi, H. Larocque, R. Balboni, G. C. Gazzadi, S. Frabboni, P.-H. Lu, E. Mafakheri, F. Bouchard, R. E. Dunin-Borkowski, R. W. Boyd, M. P. J.
Lavery, M. J. Padgett, and E. Karimi, Nat. Commun. 8, 15536 (2017).

[14] V. Grillo, T. R. Harvey, F. Venturi, J. S. Pierce, R. Balboni, F. Bouchard, G. C. Gazzadi, S. Frabboni, A. H. Tavabi, Z.-A. Li, R. E. Dunin-Borkowski, R. W. Boyd, B. J. McMorran, and E. Karimi, Nat. Commun. 8, 689 (2017).

[15] B. J. McMorran, A. Agrawal, I. M. Anderson, A. A. Herzing, H. J. Lezec, J. J. McClelland, and J. Unguris, Science 331, 192 (2011).

[16] V. Grillo, G. Carlo Gazzadi, E. Karimi, E. Mafakheri, R. W. Boyd, and S. Frabboni, Appl. Phys. Lett. 104, 043109 (2014).

[17] M. Uchida and A. Tonomura, Nature (London) 464, 737 (2010).

[18] R. Shiloh, Y. Lereah, Y. Lilach, and A. Arie, Ultramicroscopy 144, 26 (2014).

[19] A. Jesacher, S. Fürhapter, S. Bernet, and M. Ritsch-Marte, Phys. Rev. Lett. 94, 233902 (2005).

[20] S. Fürhapter, A. Jesacher, C. Maurer, S. Bernet, and M. RitschMarte, Adv. Imaging Electron Phys. 146, 1 (2007).

[21] D. Karlovets, arXiv:1803.09150.

[22] G. Pozzi, P.-H. Lu, A. H. Tavabi, M. Duchamp, and R. E. DuninBorkowski, Ultramicroscopy 181, 191 (2017).

[23] J. Verbeeck, A. Béché, K. Müller-Caspary, G. Guzzinati, M. A. Luong, and M. Den Hertog, Ultramicroscopy 190, 58 (2018).

[24] Y. Aharonov and D. Bohm, Phys. Rev. 115, 485 (1959).

[25] T. H. Boyer, Phys. Rev. D 8, 1679 (1973).

[26] A. H. Tavabi, V. Migunov, C. Dwyer, R. E. Dunin-Borkowski, and G. Pozzi, Ultramicroscopy 157, 57 (2015).

[27] V. Grillo and E. Rotunno, Ultramicroscopy 125, 97 (2013).

[28] A. M. Blackburn, Microsc. Microanal. 22, 1710 (2016). 